\title{
Certificación forestal y sustentabilidad: el caso de Malasia
}

DOI: 10.32870/mycp.v10i30.293

Antonina Ivanova*

\section{Introducción}

L a expansión de las iniciativas de desarrollo sustentable desde los años noventa es un reflejo del énfasis que se pone en las soluciones integrales al desarrollo económico, la estabilidad sociopolítica y la salud ambiental en la comunidad global. La certificación en el sector forestal promueve el desarrollo sustentable, la equidad económica y social, y en particular la responsabilidad corporativa, porque debe incluir en la toma de decisiones a los miembros de las comunidades residentes en las áreas forestales. Sin embargo, algunas veces este proceso se vuelve también contradictorio, debido a que los pequeños productores no disponen del capital necesario para la certificación y tienen dificultades en el acceso a los mercados internacionales. También en la práctica existen casos en los que los intereses comunitarios no se toman en cuenta.

La industria forestal y la certificación de madera en Malasia han sido seleccionadas como estudio de caso, dado que presentan un excelente ejemplo para ilustrar la interrelación entre la certificación y el desarrollo sustentable, así como los problemas que surgen en el proceso. Después de esta introducción, las dos siguientes partes de este artículo presentan un panorama

* Profesora-investigadora y coordinadora del Centro de Estudios de APEC, Universidad de Baja California Sur. ORCID http:// orcid.org/0000-0003-1591-6006

24 MÉXICO YLACUENCA DEL PACÍFICO

24 vol.10, núm.30 / septiembre-diciembre de 2007 del sector forestal, su importancia para la mitigación del cambio climático y la biodiversidad, así como el estatuto de la certificación de la madera a nivel global. En la tercera parte del artículo se desarrolla el caso de Malasia como el país de Asía-Pacífico con más avances en materia de certificación forestal, con sus logros y futuros retos. La conclusión presenta las posibilidades de desarrollo que ofrece la certificación de madera y los futuros retos a resolver en este ámbito.

\section{Bosques y sustentabilidad}

La industria forestal en el mundo

En los últimos 15 años la tala de madera ha sido de tres mil millones de metros cúbicos y se ha mantenido relativamente estable. Sin embargo, se estima que la cantidad real es mucho mayor, debido a la gran cantidad de tala ilegal que no se refleja en las estadísticas. Cerca del 60\% de la madera obtenida se destina a la construcción y la industria, mientras que el resto se usa como leña.

En el año 2000 las plantaciones de bosques - aun cuando representaban solamente $5 \%$ de la superficie total- proporcionaban cerca de $35 \%$ de la madera a nivel mundial, lo cual indica una tendencia a concentrar la cosecha en áreas más pequeñas. La posibilidad de satisfacer las necesidades de la sociedad por medio de 
un manejo intensivo de áreas boscosas de menor tamaño crea oportunidades para mejorar la protección de los bosques y la conservación en los demás espacios.

Los recursos forestales contribuyen de manera directa al sustento de $90 \%$ de los 1.2 millones de personas que viven en condiciones de pobreza extrema, y de manera indirecta apoyan a la agricultura y al suministro de alimentos de casi la mitad de la población del mundo en desarrollo (Banco Mundial, 2003).

Sólo unos cuantos países en vías de desarrollo se encuentran entre los mayores productores y consumidores de productos forestales, con excepción de la producción de leña (FAO, 2005). La energía generada por el consumo de madera asciende a $7-9 \%$ del total de energía consumida a nivel mundial; sin embargo, este porcentaje llega a $80-95 \%$ en algunos países en vías de desarrollo. Más de dos mil millones de personas dependen de la leña para cocinar y para calentarse. Algunas de estas personas empezarán a usar otros recursos energéticos, pero se estima que el rápido crecimiento poblacional en los países en vías de desarrollo incrementará el número de dependientes de leña en el futuro.

El incremento de la producción total de productos forestales ha resultado en una elevación del valor del comercio internacional de estos productos de 100 a 150 mil millones de dólares (mmd) entre 1993 y 2003 (Unasylva, 2004). Es importante destacar que la mayor cantidad de madera se comercializa en los mercados internos (Mersmann, 2004). Durante la década de 1990 a 2000 la creación de empleos en el sector forestal creció en cerca de $4 \%$, es decir de 12.4 a 12.9 millones de empleos (Lebedys, 2004). En el año 2000, el total del valor agregado en el sector forestal ascendió a 354 mmd (1.2\% del PIB global), mientras que casi la mitad del valor agregado se generó en la producción de papel y pulpa (Lebedys, 2004).

El panorama global del comercio de madera y productos forestales ha cambiado de manera importante en los años recientes debido a nuevos mercados emergentes como China y Rusia, así como al hecho de que los exportadores tradicionales de productos forestales primarios del sudeste asiático se transformaron en exportadores de productos procesados (Hashimoto y Moriguchi, 2004). China es ahora el mayor importador de madera industrial en el mundo (FAO, 2005).

El desarrollo de las actividades ambientales relacionadas con los bosques, como la protección de la biodiversidad, el secuestro de carbono, la protección de cuencas y el turismo alternativo están recibiendo creciente atención como instrumentos para fomentar el manejo sustentable de los bosques. Sin embargo, su expansión ha sido lenta y muy dependiente de la intervención gubernamental (Katila y Pustjärvi, 2004).

\section{Bosques, cambio climático,} biodiversidad

El manejo sustentable de los bosques puede ser caracterizado también como manejo sustentable de carbono; por lo tanto, los instrumentos que promueven el manejo sustentable de los bosques promueven a la vez la mitigación ${ }^{1}$ del cambio climático. El sector forestal puede contribuir a la reducción de las fuentes de emisión y al incremento de la absorción de emisiones. Debido a la relación entre las decisiones de uso de la tierra y el desarrollo sustentable, el sector forestal cumple un papel clave para la implementación de acciones para mitigar el cambio climático en el contexto 
más amplio del desarrollo sustentable. Como ya fue mencionado, cientos de miles de familias dependen de los bienes y servicios que proveen los bosques. Los cambios en el uso de tierra pueden afectar de manera negativa a estas familias. La deforestación continúa a ritmos alarmantes, cada año se reportan 13 millones de hectáreas deforestadas debido ante todo a la conversión de bosques en tierras agrícolas. A la vez, la deforestación aporta la mayor cantidad de las emisiones de gases con efecto invernadero debido a los cambios en el uso de la tierra. Las áreas forestales netas ${ }^{2}$ continúan disminuyendo, sin embargo, a un ritmo más bajo que en el período anterior al año 2000 , ascendiendo a 7.3 millones hectáreas por año durante el periodo 2000-2005 (UNECE/FAO, 2006a). La plantación de bosques, la restauración de paisajes y la expansión natural de los bosques han reducido la pérdida neta de áreas forestales.

Según los modelos regionales de prospectiva del impacto de las acciones en el sector forestal sobre la mitigación del cambio climático, para el año 2030 el potencial económico de la reforestación, disminución de los niveles de deforestación, manejo forestal, agricultura forestal y bioenergía $^{3}$ pueden proporcionar un descenso adicional de las emisiones en $3,150 \mathrm{MtCO}_{2}$ anualmente. Este descenso de emisiones se localizará en los trópicos y se obtendrá ante todo mediante el uso de biomasa y bioenergía (IPCC, 2004).

Las políticas orientadas a hacer las actividades forestales más sustentables han tenido mayor éxito cuando han apoyado la industria forestal para ser más lucrativa que los usos alternativos de la tierra, y cuando están respaldadas por regulaciones e instituciones que permitan llevar a cabo las medidas necesarias.
Por lo general, las actividades que se orientan hacia la conservación y mejora del secuestro de carbono son consistentes con los objetivos del manejo sustentable de los bosques (Kauppi et al., 2001). Estas actividades pueden traer beneficios adicionales como creación de empleos e ingresos adicionales, conservación de la biodiversidad y cuencas acuíferas, así como servicios de recreación.

La pérdida de los bosques es especialmente devastadora para la biodiversidad. Aunque los bosques tropicales abarcan menos de $10 \%$ de la superficie de la tierra, la mitad de las especies de flora y fauna terrestre habitan en ellos (Teck y Valencia, 1990: 16). Se espera que cerca de 10 a $20 \%$ del área forestal se convierta a otros usos para el año 2050, con consecuencias sobre el cambio climático y la biodiversidad. ${ }^{4}$ La mayor parte de esa conversión se debe a la expansión de la agricultura, seguida por la urbanización y la construcción de infraestructura (MEA, 2005).

\section{El manejo forestal y la certificación de madera: instrumento para la sustentabilidad}

\section{Esquemas internacionales \\ de certificación}

La idea sobre la certificación de madera nació a mediados de los años ochenta, con la propuesta de la delegación del Reino Unido a la Organización Internacional de Madera Tropical (ITTO, por sus siglas en inglés), la cual proponía que las prácticas de manejo forestal de los países productores de madera, sobre todo aquellos con bosques tropicales, sean evaluados en cuanto a su sustentabilidad (Crossley, 1996). Los países en vías de desarrollo de las regiones tropicales, a su vez, propusie- 
ron que la evaluación se aplicara a todos los miembros de la ITTO y que los países de bosques templados también cumplieran con los estándares de sustentabilidad y la responsabilidad ambiental a nivel global. De esta manera, los intentos para reducir la tala de bosques tropicales mediante la prohibición de importaciones y a través de extensas campañas públicas en Europa, comenzaron a sustituirse por el sistema actual de certificación independiente por parte de agencias internacionalmente reconocidas.

Se desarrollaron dos esquemas internacionales como opciones para la certificación de madera. Los "principios y criterios para la industria forestal sustentable del consejo de administración de bosques" (FSC, por sus siglas en inglés) y las "series para los sistemas de manejo ambiental 14000 de la Organización Internacional para Estandarización" (ISO, por sus siglas en inglés). El esquema de certificación de la FSC mide el estado de los bosques y la calidad del manejo de los mismos de acuerdo con estándares de desempeño prediseñados. Productos originarios de áreas forestales certificadas por la FSC y distribuidos a través de las cadenas de producción certificadas por la FSC pueden ser vendidos como productos ambientalmente amigables, o bien con etiquetas ecológicas con el logotipo de FSC. El reconocimiento de la etiqueta y la confianza del consumidor son características esenciales para los productos en los mercados certificados (Oliver, 1996). Los estándares del FSC son internacionales y se pueden aplicar a todas las regiones y tipos de bosque.

La ISO es una organización a nivel mundial que provee estándares y certificación de productos y servicios con base en convenios internacionales. Las series ISO 14000 y $14001^{5}$ evalúan la existencia de metas y el compromiso de alcanzarlas mediante un sistema de gestión ambiental en las empresas. Este tipo de evaluación no prevé evaluar el desempeño actual y no proporciona etiquetas ecológicas, aunque puede proporcionar una auditoría independiente del sistema de gestión ambiental (SGA) para uso interno (Bass, 1998).

Existen diferencias importantes entre los procesos de certificación de FSC y de ISO. El estándar de manejo ambiental de ISO (series 14000 y 14001) es un estándar de proceso que especifica cómo tiene que ser organizada la gestión de una empresa para afrontar los aspectos e impactos ambientales de sus actividades. La certificación ISO no proporciona etiqueta al producto. ISO no exige más que compromiso ambiental con legislación y regulaciones existentes, y compromiso a una mejora continua. Esto significa que hipotéticamente dos compañías forestales con diferente desempeño ambiental pudieran ambas recibir la certificación ISO. Esto también aplica a empresas madereras que funcionan en diferentes países con distinta legislación ambiental.

El esquema FSC está basado en estándares específicos de desempeño que tienen que cumplirse antes de que se emita el certificado. El sistema de acreditación de FSC está basado en los lineamientos de ISO. Es importante destacar que FSC promueve la participación de las partes involucradas; por ejemplo, recomienda que se lleve a cabo un proceso de consulta para establecer estándares regionales, y tiene que hacerse un esfuerzo especial para incluir grupos que muchas veces son excluidos del proceso de toma de decisiones. Tales grupos incluyen grupos sociales y étnicos subrepresentados, como mujeres, jóvenes, comunidades rurales, propietarios de la tierra, trabajadores forestales (FSC, 2002).

Es importante destacar que ambos

MÉXICO YLACUENCADEL PACÍFICO vol. 10, núm. 30 / septiembre-diciembre de 2007 
sistemas no se contraponen mutuamente, sino que pueden complementarse (Linden y Uusivuori, 2002). Los estándares de ISO pueden proveer el marco y los mecanismos de control para el sistema de manejo, dentro del cual los estándares de FSC servirían para medir el nivel de cumplimiento con las metas.

Programas de manejo forestal sustentable han sido establecidos en Europa, Estados Unidos y otras regiones mediante asociaciones específicas, destacando por su impacto la Asociación Americana de Bosques y Papel, la Asociación Holandesa de Comercio con Madera, el Consejo Mexicano para Industria Forestal Sustentable, el Grupo de Importación de Madera Tropical de Nueva Zelanda y el Consejo para la Industria Maderera de Malasia.

\section{Panorama del manejo forestal y la certificación de madera en el mundo}

Cerca de $90 \%$ de los bosques en los países desarrollados están administrados "de acuerdo con un plan de manejo formal o informal" (FAO, 2001). Estimaciones preliminares muestran que por lo menos 123 millones de hectáreas, cerca de $6 \%$ del área total forestal en los países en vías de desarrollo se rigen por un "plan formal de manejo forestal para un período de cinco años como mínimo, aprobado a nivel nacional" (FAO, 2001). Los planes de manejo propiamente elaborados se ven como prerrequisitos para las estrategias de desarrollo que incluyen objetivos de disminución de emisiones de carbono.

La oleada inicial de certificación de madera comenzó un poco después de la introducción del programa del $\mathrm{FSC}^{6}$ a principios de los años noventa. Para julio de 1998, un total de 10.3 millones de hectáreas de bosques fueron certificados por el FSC, de los cuales 1.5 millones correspondían a los Estados Unidos y seis millones a países europeos (cerca de $42 \%$ del área forestal de Europa) (Ingram y Enroth, 1999).

Para un periodo de 10 años el área forestal certificada anualmente por FSC se ha incrementado en $12 \%$ (FSC, 1998). Para finales del año 2000 el área certificada ascendía a 40 millones de hectáreas (FAO, 2001) y la actividad estaba en aumento.

Figura 1

Certificación de FSC

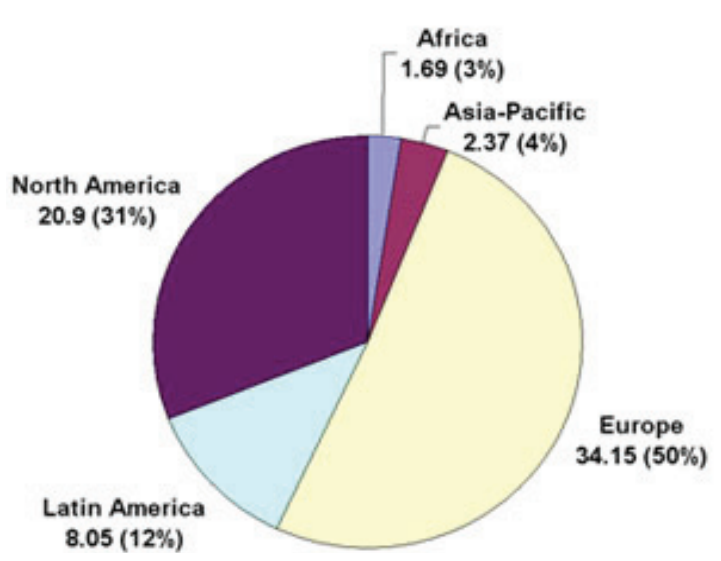

FSC-bosques certificados a fines del 2005.

Fuente: FSC (2006)

Para enero de 2006 más de 67.2 millones de hectáreas en 57 países habían sido certificadas (figura 1). Se puede observar que más del 80\% del área certificada corresponde a los países desarrollados de Europa y América del Norte (FSC, 2006). Los países de Asia-Pacífico y África, donde existen los mayores problemas de sustentabilidad y conflictos representan en conjunto sólo $7 \%$ (4\% de éstos corresponden a AsíaPacífico). Es por esto que es urgente que se emprendan programas de certificación en estas regiones. El programa desarrollado por Malasia en este ámbito sirve como ejemplo a nivel regional sobre el camino a 
Figura 2

Bosques de Asia-Pacífico

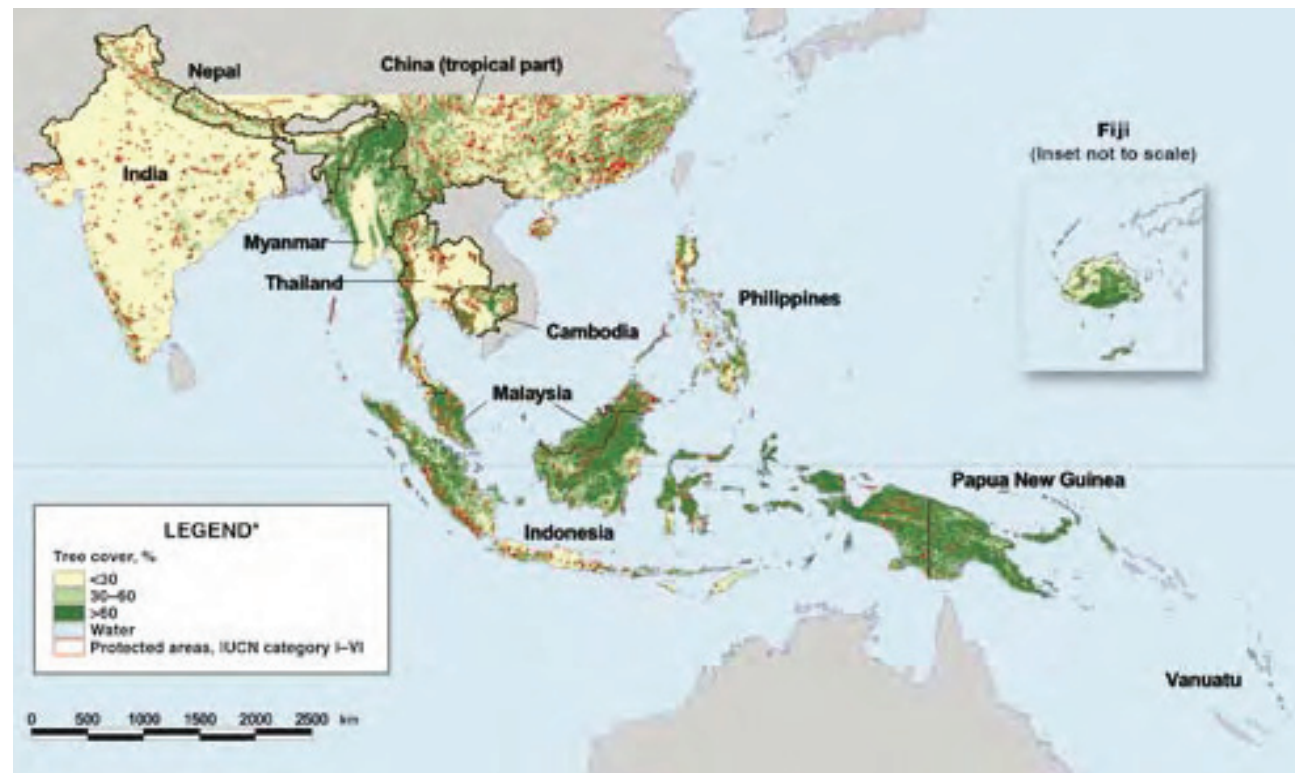

Fuente: ITTO (2006)

seguir en esta área. La madera certificada de Malasia tiene creciente demanda en los mercados internacionales y es producida en condiciones de sustentabilidad.

\section{Manejo forestal y certificación de madera en Malasia}

\section{Caracterización general}

La región Asia-Pacífico ha incrementado su producción de madera en $25 \%$ durante el periodo 1996-2000 en comparación con la década anterior (Farley, 2002). En la figura 2 se pueden apreciar las principales áreas forestales de la región. El Informe del Banco Mundial de 2003 menciona que algunas de las más importantes áreas forestales de la región podrían desaparecer en un periodo de entre tres y 10 años si no se llevan a cabo acciones a nivel gubernamental en contra de la tala ilegal y la destrucción del hábitat.
Como se puede observar en el cuadro 1 sólo $14.7 \%$ de las áreas naturales de bosques en Asia-Pacífico se manejan de manera sustentable, y sólo 5\% están certificadas. Indonesia y Papúa Nueva Guinea (dos de los principales productores de madera junto con Malasia) han certificado sólo una cantidad insignificante de sus bosques, mientras que el resto de los países en el área no tienen bosques naturales certificados (Hernández y Dewitt, 2003). En cuanto a las plantaciones de bosques, el proceso de certificación es incipiente. Malasia es el único país que ha certificado una cantidad considerable de sus bosques (41\%).

Los bosques en Malasia, en general, están manejados eficientemente, aunque existen diferencias entre Malasia peninsular, por un lado, con un control más fuerte, y por otro lado las islas de Sabah y Sarawak. El sector forestal tiene un papel muy importante en la economía de Malasia, así como en la creación de empleos. 


\section{Análisis}

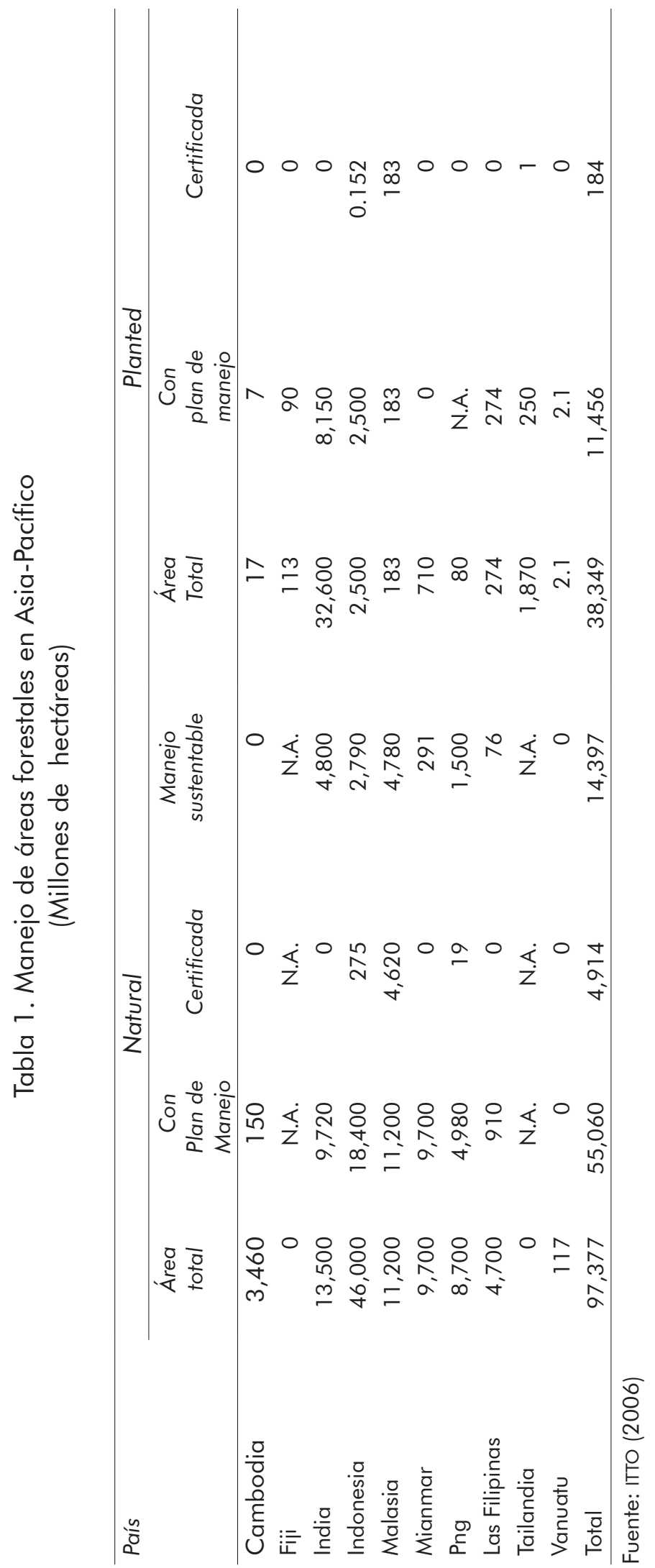


El sector produce la mayor cantidad de productos de madera con valor agregado en el mercado mundial, y continúa expandiéndose.

Gran parte de su industria maderera se basa en el caucho, que se cultiva en plantaciones. La cosecha de los bosques naturales se exporta, por lo general, como madera para la industria y la construcción. Existe administración a nivel federal y estatal que vela sobre la aplicación de las prácticas necesarias por los concesionarios y el manejo de largo plazo del recurso forestal.

Figura 3

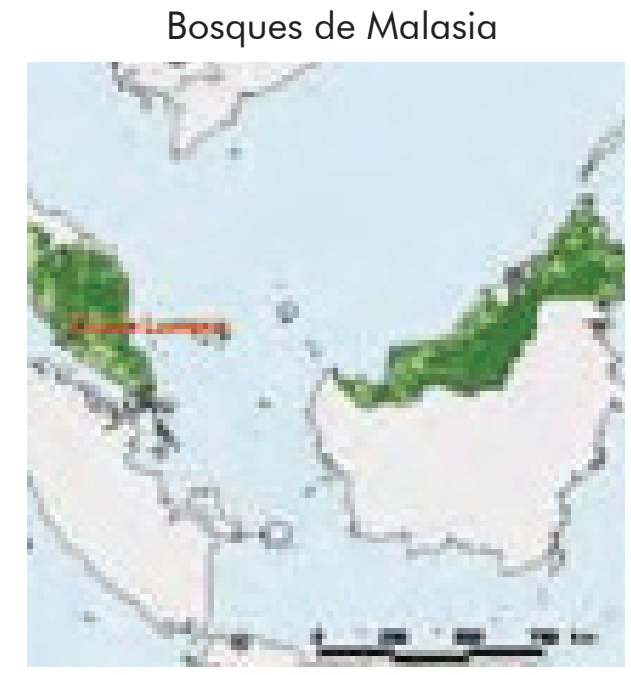

Fuente: ITTO (2006)

Las áreas forestales de Malasia (figura 3) incluyen 11.2 millones de hectáreas de bosques naturales, 183,000 hectáreas de bosques plantados y 3.21 millones de hectáreas de bosques protegidos. Se estima que cerca de 4.79 millones de hectáreas de los bosques naturales y el total de los bosques protegidos se manejan de manera sustentable.

Malasia es el mayor exportador en el mundo de madera tropical y productos de madera. Sin embargo, esta madera proviene de bosques que durante años han sido severamente sobreexplotados. Según datos de la FAO, durante los años noventa el país perdió $13.4 \%$ de su área forestal (2.7 millones de hectáreas). La tala ilegal continúa incrementando el nivel de deforestación en Malasia, el cual aumentó en $86 \%$ en el periodo 2000-2005. El país perdió en promedio 140,200 hectáreas $(0.65 \%$ de su área forestal) anualmente desde el año 2000.

Malasia está planeando seguir con la deforestación "legal" (en otras palabras, "conversión de los bosques") de otras 3.9 hectáreas. Otro aspecto a considerar es la falta de respeto hacia los derechos de los indígenas y en general las comunidades ubicadas en áreas forestales (Thang, 2003). La deforestación se lleva a cabo en localidades donde las comunidades dependen de los bosques para sobrevivir, muchas veces sin tomar en cuenta su opinión.

Desarrollo del proceso de certificación en Malasia

Malasia ha tomado decisiones importantes sobre la certificación de sus bosques y productos de Madera, de acuerdo con los objetivos de ITTO $2000{ }^{7}$

El Consejo de Certificación de Madera de Malasia (CCMM) ${ }^{8}$ se estableció en 1998, después de severas críticas de su industria forestal, que continuaron por más de 10 años. Malasia quiso reanudar sus exportaciones de madera a Europa, en donde el comercio sufrió debido a la percepción de que los productos provenían de bosques sin manejo sustentable. El papel del CCMM ha consistido en servir como esquema de certificación nacional para asegurar a los compradores que los productos de Malasia provienen de bosques sustentablemente manejados. El documento Criterios, indicadores, actividades y estándares de desempe- 
ño para la certificación del manejo forestal (MC\&I, 2002) $)^{9}$ contiene los estándares adoptados por el CCMM. Hoy en día cuatro millones de hectáreas de bosques en Malasia están certificados como "manejados de manera sustentable" por el CCMM.

A su vez, nueve unidades de manejo forestal (UMF) que abarcan un área de 4.73 millones de hectáreas han sido certificadas por la CCMM, y 83 compañías de la industria maderera han obtenido certificados de la cadena de producción de la CCMM. Actualmente el Grupo Técnico de la CCMM está desarrollando un estándar de certificación para los bosques plantados con base en los principios y criterios del FSC.

Basado en el documento Criterios para la medición del manejo sustentable de los bosques tropicales, el CCMM ha formulado un total de 92 actividades para llevar a la práctica los cinco criterios y los 27 indicadores de ITTO en cuanto al manejo forestal sustentable (MFS) a nivel nacional.

El Consejo también identificó 84 actividades para ser implementadas a nivel unidad de manejo forestal. Para diseñar las acciones fueron revisados los Principios y criterios para el manejo de bosques naturales del FSM y los criterios de la Iniciativa Alemana de Tropenwald. También se tomaron en cuenta los Lineamientos de ITTO sobre la conservación de la diversidad biológica en los bosques tropicales.

La Constitución de Malasia estipula que el sector forestal se encuentra bajo la jurisdicción de los respectivos gobiernos estatales. Así, cada estado está facultado para emitir leyes y formular políticas sectoriales de manera independiente. A su vez, las decisiones que se refieren al manejo forestal, la conservación y el desarrollo se toman a nivel estatal y las actividades se llevan a cabo por las respectivas autoridades estatales. La implementación de los criterios, indicadores y actividades se monitorean y evalúan a nivel estatal por agencias federales. En Malasia peninsular los departamentos forestales estatales también formulan criterios para el monitoreo y evaluación de las actividades forestales (Thang, 1998).

Las organizaciones no gubernamentales (ONG) ambientalistas hace tiempo han acusado a Malasia de participar en operaciones de tala ilegal en otros países, así como de introducir a su territorio dicho producto ilegal, principalmente de Indonesia. En los años recientes el país ha tomado medidas para resolver este problema a nivel doméstico, reforzando las condenas a prisión de los involucrados en la tala ilegal. También se implementaron sistemas de monitoreo a distancia para detectar las talas ilegales y los fuegos en los bosques. Por otro lado, Malasia pedirá a los países proveedores de madera a su territorio que presenten certificados de origen de la madera según el Informe de la ITTO. Esto ayudará a Malasia a responder a las acusaciones de que sus empresas madereras son cómplices en la tala ilegal en otros países.

La ITTO considera que esta decisión incrementará las ventas de productos de madera procesados de Malasia, ante 
todo a mercados donde la certificación de madera es importante (Europa y Estados Unidos).

La certificación será garantía de que Malasia no comercializa madera talada ilegalmente e introducida al territorio del país. El acuerdo de colaboración voluntaria que el país actualmente negocia con la Unión Europea es un instrumento para que los países europeos tengan seguridad de que la madera que compran proviene de países con manejo forestal sustentable (MFS). Los productores de madera alegan que los compradores europeos deben pagar más por la madera certificada, debido a que los costos de producción son más altos.

Logros del proceso de certificación forestal en Malasia

Con la implementación de MBS desde el año 2000, la tala de árboles se ha reducido de manera significativa. En el futuro la cantidad de las exportaciones de madera será determinada por la cuota de exportación. Se han diseñado estrategias a corto y mediano plazos para expandir la capacidad de procesamiento de madera. Éstas incluyen exención de impuestos para las empresas procesadoras de madera, suministro de infraestructura terrestre, creación de instalaciones de transporte marítimo y establecimiento de áreas especiales para trabajar la madera, llamadas zonas para procesamiento de madera (ZPM).

Hay un creciente número de especies de árboles que se aprovechan y comercializan de acuerdo con la demanda en los mercados internacionales. Por ejemplo, cuando se presenta una coyuntura económica de crecimiento, por lo general aumenta la construcción de viviendas y la inversión en bienes raíces, lo cual a su vez aumenta la demanda de madera.
Se ha conseguido también mayor afluencia de inversión extranjera en el sector forestal. En Sabah existe significativa participación de la inversión extranjera: $20 \%$ del total de la inversión en el sector forestal. Del total de la inversión extranjera, prevalece Taiwán con 72.96\%, Japón con $9.47 \%$, Singapur con $14.86 \%$, y Corea con 1.69\% (Thamg, 2003: 4). Sin embargo, las concesiones forestales en su totalidad pertenecen a locales.

Los bosques plantados tienen una creciente importancia para asegurar el manejo sustentable de los bosques (MSB). El gobierno ha establecido dos programas para apoyar la plantación de árboles. El primer programa es el de reforestación, implementado por el Departamento Forestal de Sarawak. El segundo programa incluye otorgamiento de licencias para la plantación de bosques al sector privado, con el objetivo de producir productos forestales.

No hay cuotas o estímulos que afecten la producción o el comercio. No existen barreras no arancelarias, excepto los requerimientos de certificación. Los empresarios privados reciben estímulos para las actividades de procesamiento de la madera. También las compañías privadas tienen incentivos para explorar el aprovechamiento de especies menos conocidas y su comercialización en el mercado internacional. El consumo interno de madera en Malasia se ha incrementado debido al desarrollo de infraestructura.

Como un esfuerzo para promover la sustentabilidad del sector forestal, se aceptó el FSC como un estándar de credibilidad para la certificación de los bosques, para mejorar el manejo forestal y para parar la tala ilegal. Este sistema también incentiva a las empresas surtir su producto de bosques bien manejados. A la fecha existen dos 
unidades de manejo forestal certificados por FSC: la Reserva Forestal Deramakot en Sabah y el Complejo Integrado de Madera Perak (CIMP) en Malasia peninsular. La Reserva Forestal Deramakot consiste de 55,083 hectáreas de bosques mixtos, y el CIMP abarca un área de 9,765 hectáreas de bosques mixtos ubicados en colinas. La certificación de FSC fue obtenida también por una plantación de caucho llamada Golden Hope Plantation, que abarca 12,434 hectáreas en el centro de Malasia peninsular.

\section{Problemas y retos a superar en la certificación forestal}

La experiencia de Malasia ha demostrado que la certificación de madera tiene altos costos. Los costos iniciales para mejorar las operaciones de cosecha se incrementan en 65.05 dólares, o en $62.5 \%$ por hectárea ${ }^{10}$ (Ahmad Fauzi et al., 2002), mientras que las ganancias a largo plazo todavía no se pueden estimar con precisión. Debido a esto, el éxito de la certificación realizada de manera voluntaria dependerá de la disposición del consumidor a pagar más (un "premio ecológico") por el producto certificado.

Mediante la certificación los consumidores podrán distinguir la madera que proviene de bosques con manejo sustentable de la madera de tiene origen en lugares con prácticas destructivas. En teoría, esto debe dar ventaja a los productores que introducen el manejo forestal sustentable por una demanda más alta y un precio más alto, que absorba los costos de la certificación. Sin embargo, en la práctica mundial todavía no todos los consumidores e importadores están dispuestos a pagar más por productos ecológicamente amigables (Counsell y Loraas, 2002).
No todos los sectores involucrados en el proceso de certificación aprueban la fuerte participación gubernamental. Sin embargo, ésta es indispensable debido a los requerimientos financieros de los esquemas de certificación, pues muchos de estos procesos todavía no pueden realizarse de manera autofinanciable (Sasser, 2003). Además en Malasia, como en muchos países en vías de desarrollo, las áreas forestales son de propiedad pública. Por otro lado, las comunidades que dependen de los bosques para su supervivencia consideran su bienestar como responsabilidad gubernamental.

Las comunidades locales de Malasia se oponen a los esfuerzos del gobierno por reverdecer la industria forestal. Durante la reunión de la Convención sobre la Biodiversidad en Malasia en febrero de 2007, representantes de 253 comunidades indígenas que habitan áreas forestales presentaron un comunicado que describe el Consejo para la Certificación de Madera de Malasia (CCMM) como un esquema que ha causado, y sigue causando, mayor marginalización de las comunidades. Sobre el documento se siguen recabando firmas de otras comunidades involucradas.

La lucha comunitaria la encabezan Joango Hutan, la Red de la Gente Indígena, así como ONG relacionadas con el sector forestal. La Declaración de las comunidades Malay concluye con un rechazo a la CCMM, y demanda cambios importantes antes de que este organismo pueda ser reconocido como un mecanismo de credibilidad para certificar el manejo sustentable de los bosques. El esquema de certificación de acuerdo con la Declaración tendrá que cumplir con los siguientes requisitos mínimos:

- Los derechos comunitarios sobre sus territorios tradicionales tienen que ser 
reconocidos.

- Tienen que ser aplicados en su totalidad los principios y los criterios del FSC, sin que sean modificados los indicadores y los verificadores mediante inserción de cláusulas que permiten que los "estándares sean sujetos a la legislación nacional existente".

- Como asesores y certificadores tienen que ser incluidos también personas de las comunidades indígenas.

- La condición más importante es que antes de que empiece la tala de árboles en tierras indígenas, deberá de obtenerse el consentimiento de las comunidades con base en previa campaña informativa y consulta popular (Thang, 2003: 8).

\section{Conclusiones}

La certificación de madera puede cumplir con dos objetivos: primero, demostrar que la madera fue obtenida en condiciones de manejo forestal sustentable, y segundo, que no es producto de prácticas ilegales. Debido a lo anterior, el producto certificado encuentra mejor aceptación en los mercados internacionales, como refleja el caso de Malasia.

La certificación de madera debe basarse en criterios e indicadores científicos y de aceptación internacional. Ésta no debe usarse como un instrumento discriminatorio para negar la entrada al mercado de los productos forestales, ante todo de los países en vías de desarrollo. Además, es necesario prestar ayuda financiera a los países en vías de desarrollo para implementar los procesos de certificación.

Los gobiernos, las organizaciones no gubernamentales, el sector empresarial y la sociedad civil tienen que participar en el proceso para asegurar que se reduzca la deforestación y que los productos y ser- vicios del sector forestal beneficien a las comunidades que más dependen de ellos. Los esquemas de certificación deben enfatizar los aspectos sociales de la producción, como salarios justos y un ambiente de trabajo digno.

La experiencia de Malasia en la certificación forestal muestra claramente la necesidad de futuras investigaciones en las siguientes áreas: a) la relación entre reformas políticas y los estándares de certificación, particularmente en el impacto de los procesos de certificación en las comunidades residentes en áreas forestales; b) cómo asegurar que los requerimientos internacionales sean relevantes y posibles de alcanzar en las condiciones específicas de cada país; c) el papel de la certificación forestal para disminuir las talas ilegales, las prácticas de corrupción y otros problemas de manejo y cumplimiento; c) cómo alcanzar los estándares de la certificación en una situación de recursos financieros restringidos.

Y finalmente, el manejo forestal sustentable, como concepto político, tanto en Malasia como en el resto del mundo debe ser parte integral de las políticas generales para el desarrollo socioeconómico y la protección ambiental. חู̣

\section{Notas}

1. Mitigación del cambio climático significa aliviar el cambio climático por medio de disminución de las emisiones de dióxido de carbono $\left(\mathrm{CO}_{2}\right)$.

2. Área forestal neta: la diferencia entre los nuevos bosques (reforestación) y la pérdida de bosques (deforestación).

3. Deforestación: pérdida de bosques. Reforestación: plantar nuevos bosques. Manejo forestal: administración y uso de los bosques para proporcionar beneficios en el tiempo. Agricultura forestal: crecimiento de bosques y cosechas agrícolas en la misma superficie. Bioenergía: uso de la biomasa como fuente de calor. Biomasa: combustible con base de cualquier organismo

MÉXICO YLACUENCADEL PACÍFICO vol. 10, núm. 30 / septiembre-diciembre de 2007 
recientemente perecido.

4. Los impactos de la deforestación sobre la vida silvestre se pueden consultar en Bass et al., 2003.

5. Consúltese List of ISO 14000 Standards en http://www.iso14000-iso14001-environmental -management.com

6. Aunque el estándar ISO 14001 ha sido adoptado, éste todavía no ha encontrado amplia aplicación en el sector forestal.

7. La Organización Internacional de Madera Tropical (ITTO) es una organización intergubernamental que promueve la conservación y el manejo sustentable, así como el aprovechamiento y el comercio relacionado con los recursos forestales tropicales. Sus 59 miembros representan cerca de $80 \%$ de los bosques tropicales en el mundo y $90 \%$ del comercio mundial con madera tropical.

8. Anteriormente su nombre fue Consejo Nacional de Malasia para Certificación de Madera.

9. Antes de adoptar los criterios se llevó a cabo un estudio-piloto sobre la certificación de madera por el Grupo de Trabajo Conjunto MalasiaHolanda, que comenzó en 1996. En el estudio participaron las UMF y las compañías exportadoras. El estudio generó también información importante en cuanto a los costos de certificación.

10. Los costos base son 472.20 dólares por hectárea (Fauzi et al., 2002).

\section{Referencias bibliográficas}

Ahmad Fauzi, P., M. Salleh, H. O. Mohd Shahwahid, N. Abdul Rahim, A. G. Awang Noor, y A. R. Mohammad Farid (2002) Cost of harvesting operations in compliance with ITTO guidelines for sustainable forest management - a model project for cost analysis to achieve sustainable forest management., Kuala Lumpur, Malasia.

Banco Mundial (2003) "Toward environmentally and socially responsible growth", Annual Report. http://www.worldbank.org/annualreport/2003/ growth.html

Bass, M., R. Aviram, y K. Parker (2003) Timber Certification: Prospects and Progress in Addressing Wildlife Issues in Central Africa, Sustainable Development and Conservation Biology Program, University of Maryland, College Park. www. Bushmeat.org/ docs.html

Counsell, S., y K. Loraas (2002) Trading in credibility: the myth and reality of the Forest Stewardship Council. Londres: Rainforest Foundation.

Crossley, R. (1996) "A Review of Global Forest Management Certification Initiatives: Political and Institutional Aspects", documento presentado en la Conferencia sobre Certificación de la UBC-OPM, mayo, Malasia.

FAO (2001) "Global Forest Resources Assessment 2000", Main Report, FAO Forestry Paper 140.

- (2005) "State of the World's Forests 2005", Annual Report.

Farley, Erin (2002) "Illegal Logging in the Asia-Pacific", The Timber Mafia. http://abc.net.au/4corners/ contents/2002/the timber_mafia/viewpoints_farley.html

FSC (1998) Certified Forests List as of 30 June 1998. Oaxaca: Forest Stewardship Council.

(2002) "FSC Principles and Criteria", FSC Document 1.2, febrero. Oaxaca: FSC.

- (2006) Certified Forests List at the Close of 2005, Forest Stewardship Council.

Hashimoto, S., y Y. Moriguchi (2004) Data Book: Material and carbon flow of harvested wood in Japan, National Institute for Environmental Studies.

Hernández, Carolina G., y David B. Dewitt (2003) Development and Security in South East Asia. Inglaterra/Estados Unidos: Ashgate Publishing Company.

Ingram, Denise, y Raija-Riitta Enroth (1999) "Timber Certification Prospects", en M. Palo, y J. Uusivuori (eds.), World Forests, Society and Environment., Col. World Forests, vol. 1, Kluwer Academic Publishers.

IPCC (2004) Good practice guidance for land use, landuse change and forestry. National Greenhouse Gas Inventories Programme. Japón: IGES.

ITTO (2002) "Forest certification: pending challenges for tropical timber", ITTO Technical Series, núm 19. Yokohama: International Tropical Timber Organization.

Katila, M., y L. Puustiärvi (2004) "Markets for forests environmental services: reality and potential", Unasylva, vol. 55, núm. 219, pp. 53-58.

Kauppi, P., R. J. Sedjo, M. Apps, C. Cerri, T. Fujimori, H. Janzen, O. Krankina, W. Makundi, G. Marland, O. Masera, G. J. Nabuurs, W. Razali, y N. H. Ravindranath (2001) "Technical and Economic Potential of Options to Enhance, Maintain and Manage Biological Carbon Reservoirs and Geoengineering. Mitigation", en B. Metz, et al. (eds.), The IPCC Third Assessment Report. Cambridge: Cambridge University Press.

Lebedys, A. (2004) Trends and current status of the contribution of the forestry sector to national economies, documento preparado para el FAO Work-Programme Component on Financing Sustainable Forest Management. Roma: FAO.

Linden, M., y J. Uusivuori (2002) "Econometric analysis of forest conservation effects: the Finnish case", Environment and Development Economics, núm. 7, pp. 281-297.

Mersmann, C. (2004) "Links between trade and sustainable forest management: an overview", Unasylva, vol. 55, núm. 219, pp. 3-9. 
Millennium Ecosystem Assessment (MEA) (2005) Ecosystems and Human Well-being: Synthesis. Washington, DC: Island Press.

Oliver, J. W. (1996) Progress in Timber Certification Initiatives Worldwide, Timber Trade Federation Forests Forever.

Sasser, E. N. (2003) "Gaining leverage: NGO influence on certification institutions in the forest products sector", en L. Teeter, B. Cashore, y D. Zhang (eds.), Forest policy for private forestry: Global and regional challenges. Oxford: CABI Publishing.

Teck, Ghee L., y Mark J. Valencia (1990) Conflict over Natural Resources in South-East Asia and the Pacific. Singapur: United Nations University Press.
Thang, H. C. (2003) Malaysia's experience in applying criteria and indicators for sustainable forest management, including forest management certification, documento presentado en la International Conference on Criteria and Indicators for Sustainable Forest Management - the Contribution of Criteria and Indicators to Sustainable Forest Management: the Way Forward, 3-7 de enero, ciudad de Guatemala.

Unasylva (2004) "Trade and Sustainable Forest Management" (editorial), vol. 55, núm. 219, p. 2.

UNECE/FAO (2006) Forest Products Annual Market Review 2005-2006. Nueva York/Ginebra: ONU. 